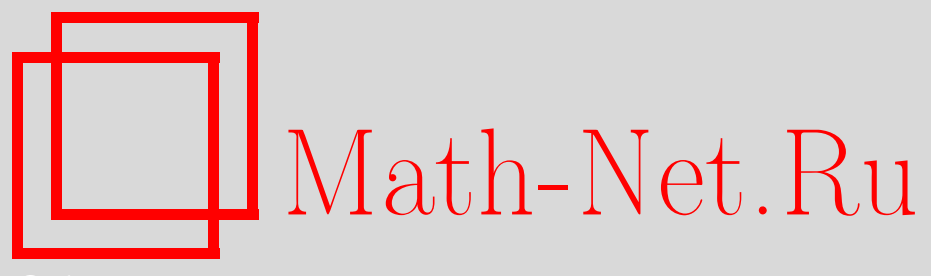

А. А. Илларионов, О среднем количестве наилучших приближений линейных форм, Изв. РАН. Сер. матем., 2014, том 78, выпуск 2, 61-86

DOI: https://doi.org/10.4213/im7978

Использование Общероссийского математического портала Math-Net.Ru подразумевает, что вы прочитали и согласны с пользовательским соглашением http://www . mathnet.ru/rus/agreement

Параметры загрузки:

IP : 54.198 .67 .100

26 апреля 2023 г., 17:05:46

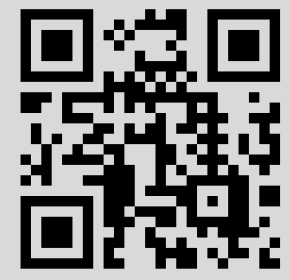


УДК $511.36+511.37$

\section{А. А. Илларионов}

\section{О среднем количестве наилучших приближений линейных форм}

Получены асимптотические формулы для среднего количества наилучших приближений линейных форм с рациональными коэффициентами и математического ожидания количества наилучших приближений линейных форм с вещественными коэффициентами.

Библиография: 14 наименований.

Ключевые слова: многомерные наилучшие приближения, $f$-наилучшие приближения линейных форм, диофантовы приближения.

DOI: $10.4213 / \mathrm{im} 7978$

\section{Введение}

Понятие наилучшего приближения играет важную роль в теории диофантовых приближений. Напомним определения.

Дробь $v / u, v \in \mathbb{Z}, u \in \mathbb{N}$, называется наилучшим приближсением (второго рода) к вещественному $\alpha$, если не существует дроби $v^{\prime} / u^{\prime}, v^{\prime} \in \mathbb{Z}, u^{\prime} \in \mathbb{N}$, такой, что

$$
\left|u^{\prime} \alpha-v^{\prime}\right| \leqslant|u \alpha-v|, \quad u^{\prime} \leqslant u,
$$

причем хотя бы одно из неравенств является строгим. Согласно классической теореме Лагранжа множество наилучших приближений к $\alpha \in(0,1 / 2)$ состоит из подходящих дробей к $\alpha$ и поэтому однозначно определяется разложением $\alpha$ в непрерывную дробь. Рассмотрим следующее обобщение понятия наилучшего приближения в многомерном случае.

Пусть функция $f: \mathbb{R}^{n} \rightarrow[0,+\infty)$ является лучевой, т. е. для всех $x \in \mathbb{R}^{n}$, $\lambda \in \mathbb{R}:$

1) $0 \leqslant f(x), f(x)=0 \Longleftrightarrow x=0$;

2) $f(\lambda x)=|\lambda| f(x)$.

Ненулевой вектор $(u, v) \in \mathbb{Z}^{n} \times \mathbb{Z}$ будем называть $f$-наилучиим приближением линейной формы $L: \mathbb{R}^{n} \rightarrow \mathbb{R}$, если не существует ненулевого вектора $\left(u^{\prime}, v^{\prime}\right) \in \mathbb{Z}^{n} \times \mathbb{Z}$ такого, что

$$
\left|L u^{\prime}-v^{\prime}\right| \leqslant|L u-v|, \quad f\left(u^{\prime}\right) \leqslant f(u),
$$

причем хотя бы одно из неравенств строгое.

Далее всюду считаем, что функция $f$ непрерывная и кусочно дифференцируемая.

Работа выполнена при финансовой поддержке РФФИ (грант № 11-01-00628-а), Президиума ДВО РАН (проект № 12-I-П19-01). 
Пусть $\mathfrak{B}_{f}(\alpha)$ - множество $f$-наилучших приближений линейной формы $x \in \mathbb{R}^{n} \rightarrow \alpha_{1} x_{1}+\cdots+\alpha_{n} x_{n}$. Множество $\mathfrak{B}_{f}(\alpha)$ конечно только в том случае, когда числа $\alpha_{1}, \ldots, \alpha_{n}, 1$ линейно зависимы над кольцом $\mathbb{Z}$.

Для любых $P \geqslant 1$ и $\alpha \in[0,1)^{n}$ определим множество

$$
\mathfrak{B}_{f}(\alpha, P)=\left\{(u, v) \in \mathfrak{B}_{f}(\alpha): f(u) \leqslant P\right\} .
$$

Оно конечно, причем

$$
\# \mathfrak{B}_{f}(\alpha, P) \underset{f}{\ll} \ln P+1 .
$$

Здесь и далее $\# X$ - количество элементов конечного множества $X$. Если $f(x)=$ $\max \left\{\left|x_{1}\right|, \ldots,\left|x_{n}\right|\right\}$, то неравенство (0.1) вытекает из известных результатов об экспоненциальном росте наилучших приближений [1, лемма 1]. В общем случае оно является тривиальным следствием из [2, теорема 1] (соотношение (0.1) также легко получается методами из [1, лемма 1]).

Для любого вещественного $R>1$ определим множество $\Delta_{n}(R)$, состоящее из рациональных векторов $\alpha=\left(\alpha_{1}, \ldots, \alpha_{n}\right), \alpha_{i}=P_{i} / Q$, где $P_{i}, Q$ целые, причем

$$
0 \leqslant P_{i}<Q \leqslant R, \quad i=\overline{1, n} \text {. }
$$

Используя теорему Минковского о выпуклом теле, получаем (см. лемму 3.1), что если $\alpha \in \Delta_{n}(R)$, то для любого наилучшего приближения $(u, v) \in \mathfrak{B}_{f}(\alpha)$ при $v \neq \alpha_{1} u_{1}+\cdots+\alpha_{n} u_{n}$ имеет место оценка

$$
f(u) \leqslant C_{f}^{*} R^{1 / n},
$$

где постоянная $C_{f}^{*}$ зависит только от $f$.

Пусть

$$
\begin{gathered}
E_{f}(R, P)=\frac{1}{\# \Delta_{n}(R)} \sum_{\alpha \in \Delta_{n}(R)} \# \mathfrak{B}_{f}(\alpha, P), \\
E_{f}(R)=\frac{1}{\# \Delta_{n}(R)} \sum_{\alpha \in \Delta_{n}(R)} \# \mathfrak{B}_{f}(\alpha)
\end{gathered}
$$

- средние количества $f$-наилучших приближений линейных форм с рациональными коэффициентами из $\Delta_{n}(R)$;

$$
\mathcal{E}_{f}(P)=\int_{[0,1)^{n}} \# \mathfrak{B}_{f}(\alpha, P) d \alpha
$$

- математическое ожидание количества $f$-наилучших приближений $(u, v)$ при $f(u) \leqslant P$. Отметим, что функция $\alpha \in[0,1)^{n} \rightarrow \# \mathfrak{B}_{f}(\alpha, P)$ измерима по Лебегу (см. лемму 3.2 ) и является ограниченной (согласно (0.1)). Поэтому интеграл Лебега $\mathcal{E}_{f}(P)$ существует и конечен.

В одномерном случае (т. е. при $n=1, f(x)=|x|$ ) асимптотические формулы для определенных средних величин вытекают из известных статистических свойств непрерывных дробей (см. [3]-[6] и ссылки в [6]). В частности,

$$
E_{f}(R)=C_{1} \ln R+O(1), \quad \mathcal{E}_{f}(P)=C_{1} \ln P+O(1), \quad C_{1}=\frac{24 \ln 2}{\pi^{2}} .
$$


Из [3], [5], [6] также следуют асимптотические формулы с двумя значащими членами и степенным понижением в остатках.

В настоящей работе мы доказываем обобщение этих результатов в многомерном случае: для любого $n \geqslant 1$ и любой непрерьвной кусочно дифференцируемой лучевой функиии $f: \mathbb{R}^{n} \rightarrow \mathbb{R}$ справедливъ формуль

$$
\begin{gathered}
E_{f}(R, P)=\mathcal{C}_{f} \ln P+O_{f}(1), \quad P \leqslant C_{f}^{*} R^{1 / n}, \\
\mathcal{E}_{f}(P)=\mathcal{C}_{f} \ln P+O_{f}(1),
\end{gathered}
$$

где $\mathcal{C}_{f}$ - положительная постоянная, зависящая толъко от $f$. Формула для $\mathcal{C}_{f}$ будет приведена ниже (см. (3.2)).

ЗАмечаниЕ 0.1. Для любого $\alpha \in[0,1)^{n}$ количество $(u, v) \in \mathfrak{B}_{f}(\alpha)$, удовлетворяющих условию $v=\alpha_{1} u_{1}+\cdots+\alpha_{n} u_{n}$, оценивается величиной $O_{f}(1)$. Поэтому, используя $(0.2)$ и выбирая $P=C_{f}^{*} R^{1 / n}$ в $(0.3)$, получаем

$$
E_{f}(R)=\frac{\mathcal{C}_{f}}{n} \ln R+O_{f}(1) .
$$

Доказательство (0.3) основано на идеях из работы [7], в которой получена асимптотическая формула для среднего числа цилиндрических минимумов целочисленных решеток. Метод из [7] позволяет свести вычисление суммы $\sum_{\alpha \in \Delta_{n}(R)} \# \mathfrak{B}_{f}(\alpha, P)$ к нахождению количества целочисленных матриц $M$ размера $(n+1) \times(n+1)$, удовлетворяющих условиям

$$
M \in \Omega_{f}, \quad|\operatorname{det} M| \in[1, R], \quad d_{n}(M)=1, \quad f\left(m_{11}, \ldots, m_{n 1}\right) \leqslant P,
$$

где $\Omega_{f}$ - некоторое подмножество в $\mathrm{GL}_{n+1}(\mathbb{R})$, а $d_{n}(M)$ - наибольший общий делитель алгебраических дополнений к элементам последней строки матрицы $M$.

Формула (0.4) получается предельным переходом в $(0.3)$ при $R \rightarrow \infty$.

В $\S 1$ мы получим асимптотическую формулу для количества целочисленных матриц, удовлетворяющих условиям вида (0.5), в 2 приведем результаты о цилиндрических минимумах и в $\S 3$ докажем формулы $(0.3),(0.4)$.

Введем следующие обозначения: $\mathbb{R}_{+}-$множество положительных вещественных чисел; $\mathbb{Z}_{+}$- множество неотрицательных целых чисел; $\partial U$ - граница множества $U$; mes - мера Лебега; $\zeta(s)$ - дзета-функция Римана; $\mathrm{GL}_{s}(\mathbb{R})$ - множество невырожденных вещественных матриц размера $s \times s ; \mathrm{SL}_{s}(\mathbb{Z})$ - множество целочисленных унимодулярных матриц размера $s \times s ; \mathcal{D}_{s}^{\prime}\left(\mathbb{R}_{+}\right)$- множество диагональных матриц из $\mathrm{GL}_{s}(\mathbb{R})$ с главной диагональю вида $\rho, \rho, \ldots, \rho, h$, где $\rho, h \in \mathbb{R}_{+}$.

Если $x=\left(x_{1}, \ldots, x_{s}\right) \in \mathbb{R}^{s}$, то

$$
|x|_{\infty}=\max _{1 \leqslant i \leqslant s}\left|x_{i}\right|, \quad|x|=\sqrt{\sum_{i=1}^{s} x_{i}^{2}}, \quad \rho(x)=\sqrt{\sum_{i=1}^{s-1} x_{i}^{2}} .
$$

Если $X=\left(\left(x_{i j}\right)\right)$ - вещественная матрица размера $s \times s$, то

$$
h_{j}(X)=x_{s j}, \quad \rho_{j}(X)=\sqrt{\sum_{i=1}^{s-1} x_{i j}^{2}}, \quad j=\overline{1, s} .
$$


Если $M$ - целочисленная матрица, то $d_{t}(M)$ - наибольший общий делитель миноров порядка $t$, составленных из элементов первых $t$ строк матрицы $M$.

Запись

$$
f(x) \ll g(x) \quad \text { (либо } f(x)=O(g(x))) \quad \text { при } x \in X
$$

означает, что существует абсолютная постоянная $C>0$ такая, что $|f(x)| \leqslant$ $C g(x)$ при всех $x \in X$. Если постоянная $C$ зависит от параметра $\theta$, то записываем $f(x) \underset{\theta}{\ll} g(x)$ (либо $f(x)=O_{\theta}(g(x))$ ). Запись $f \asymp g$ означает, что $f \ll g \ll f$.

Границу $\partial \Omega$ множества $\Omega \subset \mathbb{R}^{s}$ называем кусочно дифберенцируемой, если она состоит из фиксированного числа гиперповерхностей класса $C^{1}$.

Автор благодарен рецензенту за указанные опечатки и неточности в первоначальной версии статьи.

\section{§ 1. Количество целочисленных матриц, удовлетворяющих $(0.5)$}

Определим целое $s=n+1$. Пусть множество $\Omega \subset \mathrm{GL}_{s}(\mathbb{R})$ удовлетворяет следующим условиям:

i) $\Omega$ инвариантно относительно левого действия группы $\mathcal{D}_{s}^{\prime}\left(\mathbb{R}_{+}\right)$;

ii) существует такая постоянная $C=C(\Omega)>0$, что для любой матрицы $X \in \Omega$

$$
\begin{gathered}
\max _{1 \leqslant j \leqslant s}\left|h_{j}(X)\right| \leqslant C h_{1}(X), \quad \rho_{j}(X) \leqslant C \rho_{j+1}(X), \quad j=\overline{1, s-1}, \\
h_{1}(X) \prod_{j=2}^{s} \rho_{j}(X) \leqslant C|\operatorname{det} X|
\end{gathered}
$$

iii) граница $\partial \Omega$ кусочно дифференцируема;

iv) если $r \in[1,+\infty), Q$ - целочисленная матрица из $\mathrm{GL}_{s}(\mathbb{R})$, то количество матриц $M \in \Omega \cap\left(Q \cdot \mathrm{SL}_{s}(\mathbb{Z})\right)$, удовлетворяющих условию

$$
h_{1}(M) \leqslant r \quad \text { либо } \rho_{1}(M) \leqslant r,
$$

не больше $O_{\Omega}(\ln r+1)$.

Целью в настоящем параграфе является вывод асимптотической формулы для количества целочисленных матриц $M \in \Omega$, удовлетворяющих условиям

$$
d_{s-1}(M)=1, \quad|\operatorname{det} M| \in[1, R], \quad \rho_{s}(M) \leqslant P,
$$

где $R, P \in(1,+\infty)$. Поскольку нас интересует только главный член, доказательство будет проведено элементарными методами.

Пусть $\Omega_{R}$ - множество матриц $X \in \Omega$ таких, что

$$
|\operatorname{det} X| \in[1, R], \quad h_{1}(X) \geqslant 1, \quad \rho_{2}(X) \geqslant 1 .
$$

Определим

$$
\Omega_{R, P}=\left\{X \in \Omega_{R}: \rho_{s}(X) \leqslant P\right\} .
$$


Тогда наша задача заключается в вычислении количества целочисленных точек $M \in \Omega_{P, R}$, удовлетворяющих условию $d_{s-1}(M)=1$.

Отметим, что согласно условиям i), ii)

$$
C_{0} \equiv \inf _{X \in \Omega_{R, P}} \rho_{s}(X) \asymp 1 .
$$

1.1. Количество целочисленных точек в заданном множестве. Не претендуя на оригинальность, приведем некоторые элементарные результаты о количестве целочисленных точек в заданном множестве пространства $\mathbb{R}^{s}$.

Для любого числа $\varepsilon \in \mathbb{R}_{+}$и множества $G \subset \mathbb{R}^{s}$ определим

$$
\begin{gathered}
U(G, \varepsilon)=\left\{x \in \mathbb{R}^{s}: \rho_{\infty}(G, x) \leqslant \varepsilon\right\}, \\
B(G, \varepsilon)=G \cap U(\partial G, s \varepsilon)=\left\{x \in G: \rho_{\infty}(x, \partial G) \leqslant s \varepsilon\right\},
\end{gathered}
$$

где

$$
\rho_{\infty}(G, x)=\inf _{y \in G}|x-y|_{\infty}
$$

т. е. $U(G, \varepsilon)$ - замыкание окрестности радиуса $\varepsilon$ множества $G$, а $B(G, \varepsilon)$ состоит из точек множества $G$, которые находятся на расстоянии не более чем $s \varepsilon$ от границы $\partial G$ (относительно метрики $\left.\rho_{\infty}\right)$.

Лемма 1.1. Пусть $G$ - ограниченное измеримое по Лебегу множество из $\mathbb{R}^{s}$. Тогда

$$
\begin{gathered}
\left|\#\left(\mathbb{Z}^{s} \cap G\right)-\operatorname{mes} G\right| \leqslant \operatorname{mes} U(\partial G, 1), \\
\left|\#\left(\mathbb{Z}^{s} \cap G\right)-\operatorname{mes} G\right| \leqslant \#\left(\mathbb{Z}^{s} \cap U(\partial G, 1)\right) .
\end{gathered}
$$

ДокАзАТЕльство. Для любого целочисленного вектора $x \in \mathbb{Z}^{s}$ обозначим через

$$
\Pi(x)=[0,1)^{s}+x=\left\{y+x: y \in[0,1)^{s}\right\}
$$

сдвиг куба $[0,1)^{s}$ на вектор $x$. Отметим, что $\Pi(x) \cap \mathbb{Z}^{s}=\{x\}$. Пусть $G_{*}-$ объединение всех $\Pi(x)$, которые содержатся в $G$, а $G^{*}$ - объединение всех $\Pi(x)$, которые пересекаются с $G$, т. е.

$$
G_{*}=\bigcup_{x \in \mathbb{Z}^{s}: \Pi(x) \subset G} \Pi(x), \quad G^{*}=\bigcup_{x \in \mathbb{Z}^{s}: \Pi(x) \cap G \neq \varnothing} \Pi(x) .
$$

Тогда $G_{*} \subset G \subset G^{*}, \operatorname{mes} G_{*}=\#\left(\mathbb{Z}^{s} \cap G_{*}\right)$, mes $G^{*}=\#\left(\mathbb{Z}^{s} \cap G^{*}\right)$. Поэтому

$$
\left|\operatorname{mes} G-\#\left(G \cap \mathbb{Z}^{s}\right)\right| \leqslant \operatorname{mes}\left(G^{*} \backslash G_{*}\right)=\#\left(\mathbb{Z}^{s} \cap\left(G^{*} \backslash G_{*}\right)\right) \text {. }
$$

Осталось учесть, что $G^{*} \backslash G_{*} \subset U(G, 1)$.

Лемма 1.2. Пусть $G$ - ограниченное измеримое по Лебегу множество из $\mathbb{R}^{s}$. Пусть Г- полная целочисленная решетка в $\mathbb{R}^{s}, \operatorname{det} \Gamma=D$. Тогда

$$
\#(\Gamma \cap G)=\frac{\#\left(\mathbb{Z}^{s} \cap G\right)}{D}+O\left(\xi_{1}+\frac{\xi_{2}}{D}\right),
$$

где $\xi_{1}=\#(\Gamma \cap B(G, 2 D)), \xi_{2}=\#\left(\mathbb{Z}^{s} \cap B(G, 2 D)\right)$. 
ДокАЗАТЕЛЬство. Пусть $\left\{e^{(i)}\right\}_{i=1}^{s}-$ базис $Г$, удовлетворяющий условиям $\left|e^{(i)}\right|_{\infty} \leqslant D$. Такой базис всегда существует (см., например, [8, гл. 1]). Обозначим через

$$
\widetilde{\Pi}=\left\{t_{1} e^{(1)}+\cdots+t_{s} e^{(s)}: t_{i} \in[0,1)\right\}
$$

основной параллелепипед Г и определим $\widetilde{\Pi}(\gamma)=\{x+\gamma: x \in \widetilde{\Pi}\}$.

Пусть $G^{\prime}$ - объединение всех $\widetilde{\Pi}(\gamma)$ таких, что

$$
\gamma \in \Gamma, \quad \widetilde{\Pi}(\gamma) \subset G, \quad \rho_{\infty}(\partial G, \widetilde{\Pi}(\gamma))>1 .
$$

Тогда $G \backslash G^{\prime} \subset B(G, 2 D), U\left(\partial G^{\prime}, 1\right) \subset B(G, 2 D)$. Поэтому, используя (1.3) для выражения mes $G^{\prime}$ через число целых точек в $G^{\prime}$, получаем

$$
\begin{aligned}
& \#(\Gamma \cap G)=\#\left(\Gamma \cap G^{\prime}\right)+O\left(\xi_{1}\right)=\frac{\operatorname{mes} G^{\prime}}{D}+O\left(\xi_{1}\right) \\
& \quad=\frac{\#\left(\mathbb{Z}^{s} \cap G^{\prime}\right)+O\left(\#\left(\mathbb{Z}^{s} \cap U\left(\partial G^{\prime}, 1\right)\right)\right)}{D}+O\left(\xi_{1}\right)=\frac{\#\left(\mathbb{Z}^{s} \cap G\right)+O\left(\xi_{2}\right)}{D}+O\left(\xi_{1}\right) .
\end{aligned}
$$

Лемма доказана.

1.2. Количество целочисленных матриц из $\Omega_{R, P}$. Вычислим количество целочисленных матриц из $\Omega_{R, P}$, используя формулу (1.2).

Согласно условиям i)-iii) множество $\Omega$ можно представить в виде

$$
\Omega=D_{s}^{\prime}\left(\mathbb{R}_{+}\right) \cdot \Omega_{0}=\left\{\operatorname{Diag}(\rho, h) \cdot M: M \in \Omega_{0}, \rho, h \in \mathbb{R}_{+}\right\},
$$

где $\operatorname{Diag}(\rho, h)$ - матрица из $\mathcal{D}_{s}^{\prime}\left(\mathbb{R}_{+}\right)$с главной диагональю $\rho, \rho, \ldots, \rho, h$, а $\Omega_{0}-$ ограниченное множество с кусочно дифференцируемой границей, лежащее на поверхности

$$
\mathcal{S}=\left\{X \in \mathrm{GL}_{s}(\mathbb{R}): h_{1}(X)=\rho_{s}(X)=1\right\}
$$

Определим

$$
\mu(\Omega)=\int_{\Omega_{0}} \frac{d \mathcal{S}(X)}{|\operatorname{det} X|^{s}},
$$

где $d \mathcal{S}(X)$ - дифференциал $\left(s^{2}-2\right)$-мерной меры Лебега поверхности $\mathcal{S}$ в точке $X$. В [7] доказано, что интеграл $\mu(\Omega)$ сходится, причем количество целочисленных матриц $M \in \Omega_{R}$ равно

$$
\frac{R^{s}}{s(s-1)}\left(\mu(\Omega) \ln R+O_{\Omega}(1)\right) .
$$

Пусть $\varepsilon, r \in[1,+\infty]$. Следующие оценки доказываются точно так же, как в [7, следствие 2, теорема 2]:

$$
\begin{gathered}
\operatorname{mes} U\left(\partial \Omega_{R, P}, \varepsilon\right) \underset{\Omega}{\ll} \varepsilon R^{s}, \\
\operatorname{mes}\left\{X \in U\left(\partial \Omega_{R, P}, \varepsilon\right): \min \left\{\rho_{1}(X),\left|h_{1}(X)\right|\right\} \geqslant r\right\} \underset{\Omega}{\ll} \frac{\varepsilon R^{s}}{r} .
\end{gathered}
$$


Лемма 1.3. Пусть $P, R \in[1,+\infty), C_{P, R}=\max \left\{1, \ln \left(P^{s-1} / R\right)\right\}$. Тогдаколичество иелочисленных матрич в $\Omega_{R, P}$ равно

$$
\frac{R^{s}}{s}\left(\mu(\Omega) \ln P+O_{\Omega}\left(C_{P, R}\right)\right)
$$

ДокАзАТЕЛЬство. Согласно неравенству (1.2) и оценке (1.4), в которой $\varepsilon=1$, количество целочисленных точек в $\Omega_{R, P}$ равно

$$
\operatorname{mes} \Omega_{R, P}+O_{\Omega}\left(R^{s}\right) \text {. }
$$

Осталось вычислить mes $\Omega_{R, P}$. Для этого мы точно так же, как и в $[7$, доказательство теоремы 2], сделаем в интеграле

$$
\operatorname{mes} \Omega_{R, P}=\int_{\Omega_{R, P}} d X
$$

замену $X=\operatorname{Diag}(\rho, h) \cdot X^{\prime}$, где $\rho=\rho_{s}(X), h=h_{1}(X)$ и $X^{\prime} \in \mathcal{S}$. Тогда

$$
d X=\left(\rho^{s-1}\right)^{s-1} \rho^{s-2} h^{s-1} d \rho d h d \mathcal{S}\left(X^{\prime}\right)
$$

и, учитывая, что

$$
|\operatorname{det} X| \in[1, R] \quad \Longleftrightarrow \quad \frac{1}{\left|\operatorname{det} X^{\prime}\right|} \leqslant \rho^{s-1} h \leqslant \frac{R}{\left|\operatorname{det} X^{\prime}\right|},
$$

получаем

$$
\operatorname{mes} \Omega_{R, P}=\int_{\Omega_{0}}\left(\int_{Q\left(X^{\prime}\right)} \rho^{s(s-1)-1} h^{s-1} d \rho d h\right) d \mathcal{S}\left(X^{\prime}\right),
$$

где

$$
Q\left(X^{\prime}\right)=\left\{(\rho, h) \in\left[C_{0}, P\right] \times[1,+\infty): \frac{1}{\left|\operatorname{det} X^{\prime}\right|} \leqslant \rho^{s-1} h \leqslant \frac{R}{\left|\operatorname{det} X^{\prime}\right|}\right\},
$$

а $C_{0}$ - постоянная из (1.1). Поскольку

$$
\begin{gathered}
\int_{Q\left(X^{\prime}\right)} \rho^{s(s-1)-1} h^{s-1} d \rho d h=\frac{R^{s}}{s} \frac{\ln P}{\left|\operatorname{det} X^{\prime}\right|^{s}}+O_{s, C_{0}}\left(C_{P, R} R^{s} \frac{1+|\ln | \operatorname{det} X^{\prime}||}{\left|\operatorname{det} X^{\prime}\right|^{s}}\right), \\
\int_{\Omega_{0}} \frac{d \mathcal{S}\left(X^{\prime}\right)}{\left|\operatorname{det} X^{\prime}\right|^{s}}=\mu(\Omega), \quad \int_{\Omega_{0}} \frac{1+|\ln | \operatorname{det} X^{\prime}||}{\left|\operatorname{det} X^{\prime}\right|^{s}} d \mathcal{S}\left(X^{\prime}\right)<+\infty
\end{gathered}
$$

Tо

$$
\operatorname{mes} \Omega_{R, P}=\frac{R^{s}}{s}\left(\mu(\Omega) \ln P+O_{\Omega}\left(C_{P, R}\right)\right) \text {. }
$$

Лемма доказана. 
1.3. О количестве целочисленных матриц $M$ с $d_{t}(M)=1$. Пусть $\mathrm{M}_{s}(\mathbb{R})$ - множество вещественных матриц размера $s \times s$.

Лемма 1.4. Пусть $\Theta$ - ограниченное измеримое по Лебегу множество из $\mathrm{M}_{s}(\mathbb{R})$, а $\Theta(\mathbb{Z})$ - множество иелочисленньх матрии, из $\Theta$. Определим

$$
\begin{gathered}
g(0)=\# \Theta(\mathbb{Z}), \\
g(t)=\#\left\{M \in \Theta(\mathbb{Z}): d_{t}(M)=1\right\}, \quad t=\overline{1, s-1 .}
\end{gathered}
$$

Тогда для любого $t \in\{0, \ldots, s-2\}$ имеет место формула

$$
g(t+1)=\frac{1}{\zeta(s-t)} g(t)+O\left(\sum_{k \in \mathbb{N}}\left(\xi_{1}(k)+\frac{\xi_{2}(k)}{k^{s-t}}\right)\right),
$$

где $\xi_{1}(k)$ - количество иелочисленных матрии, $M \in B\left(\Theta, 2 k^{s-t}\right)$ таких, что

$$
d_{t}(M)=1, \quad d_{t+1}(M) \equiv 0(\bmod k),
$$

а $\xi_{2}(k)$ - количество иелочисленных матрии, $M \in B\left(\Theta, 2 k^{s-t}\right)$ таких, что $d_{t}(M)=1$.

ДокАЗАТЕЛЬСтво. Если $d_{t+1}(M)=1$, то и $d_{t}(M)=1$. Поэтому, используя свойства функции Мёбиуса $\mu$, получаем

$$
g(t+1)=\sum_{k \in \mathbb{N}} \mu(k) g(t, k)
$$

где $g(t, k)$ - количество матриц $M \in \Theta(\mathbb{Z})$, удовлетворяющих (1.6).

Пусть множество $\Theta^{\prime}$ составлено из матриц, полученных из $X \in \Theta$ вычеркиванием $(t+1)$-й строки. Возьмем $X^{\prime} \in \Theta^{\prime}$ и $x \in \mathbb{R}^{s}$. Между $t$-й и $(t+1)$-й строками матрицы $X^{\prime}$ вставим $x$ в качестве еще одной строки и обозначим полученную матрицу через $\mathcal{M}\left(X^{\prime}, x\right) \in \mathrm{M}_{s}(\mathbb{R})$, т. е.

$$
\mathcal{M}\left(X^{\prime}, x\right)=\left(\begin{array}{ccccc}
x_{11}^{\prime} & x_{12}^{\prime} & x_{13}^{\prime} & \ldots & x_{1 s}^{\prime} \\
\ldots & \ldots & \ldots & \ldots & \ldots \\
x_{t 1}^{\prime} & x_{t 2}^{\prime} & x_{t 3}^{\prime} & \ldots & x_{t s}^{\prime} \\
x_{1} & x_{2} & x_{3} & \ldots & x_{s} \\
x_{(t+1) 1}^{\prime} & x_{(t+1) 2}^{\prime} & x_{(t+1) 3}^{\prime} & \ldots & x_{(t+1) s}^{\prime} \\
\ldots & \ldots & \ldots & \ldots & \ldots \\
x_{(s-1) 1}^{\prime} & x_{(s-1) 2}^{\prime} & x_{(s-1) 3}^{\prime} & \ldots & x_{(s-1) s}^{\prime}
\end{array}\right) .
$$

Пусть $G\left(X^{\prime}\right)=\left\{x \in \mathbb{R}^{s}: \mathcal{M}\left(X^{\prime}, x\right) \in \Theta\right\}$. Тогда

$$
\begin{aligned}
\Theta & =\left\{\mathcal{M}\left(X^{\prime}, x\right): X^{\prime} \in \Theta^{\prime}, x \in G\left(X^{\prime}\right)\right\}, \\
g(t, k) & =\sum_{M^{\prime} \in \Theta^{\prime}(\mathbb{Z}), d_{t}\left(M^{\prime}\right)=1} \#\left(\Gamma\left(M^{\prime}, k\right) \cap G\left(M^{\prime}\right)\right),
\end{aligned}
$$

где $\Gamma\left(M^{\prime}, k\right)$ - решетка, состоящая из решений $m \in \mathbb{Z}^{s}$ сравнения

$$
d_{t+1}\left(\mathcal{M}\left(M^{\prime}, m\right)\right) \equiv 0(\bmod k) .
$$


Поскольку $d_{t}\left(M^{\prime}\right)=1$, то $\operatorname{det} \Gamma\left(M^{\prime}, k\right)=k^{s-t}$ (см., например, [9, лемма 8]). Поэтому, применяя лемму 1.2, получаем

$$
\#\left(\Gamma\left(M^{\prime}, k\right) \cap G\left(M^{\prime}\right)\right)=\frac{1}{k^{s-t}} \#\left(\mathbb{Z}^{s} \cap G\left(M^{\prime}\right)\right)+O\left(\eta_{1}\left(M^{\prime}, k\right)+\frac{\eta_{2}\left(M^{\prime}, k\right)}{k^{s-t}}\right),
$$

где $\eta_{1}\left(M^{\prime}, k\right)$ - количество узлов решетки $\Gamma^{\prime}\left(M^{\prime}, k\right)$ в $B\left(G\left(M^{\prime}\right), 2 k^{s-t}\right)$, а $\eta_{2}\left(M^{\prime}, k\right)$ - количество целочисленных точек в $B\left(G\left(M^{\prime}\right), 2 k^{s-t}\right)$.

Если $X=\mathcal{M}\left(X^{\prime}, x\right)$, причем $\rho_{\infty}\left(x, \partial G\left(X^{\prime}\right)\right) \leqslant r$, то $\rho_{\infty}(X, \partial \Theta) \leqslant r$. Поэтому

$$
\sum_{M^{\prime} \in \Theta^{\prime}(\mathbb{Z}), d_{t}\left(M^{\prime}\right)=1} \eta_{i}\left(M^{\prime}, k\right) \leqslant \xi_{i}(k), \quad i=1,2 .
$$

Кроме того,

$$
\sum_{M^{\prime} \in \Theta^{\prime}(\mathbb{Z}), d_{t}\left(M^{\prime}\right)=1} \#\left(\mathbb{Z}^{s} \cap G\left(M^{\prime}\right)\right)=g(t) .
$$

Используя (1.7)-(1.10), получаем

$$
\begin{aligned}
g(t+1) & =\sum_{k \in \mathbb{N}} \mu(k) \sum_{\substack{M^{\prime} \in \Theta^{\prime}(\mathbb{Z}) \\
d_{t}\left(M^{\prime}\right)=1}} \#\left(\Gamma\left(M^{\prime}, k\right) \cap G\left(M^{\prime}\right)\right) \\
& =\sum_{k \in \mathbb{N}} \mu(k)\left(\frac{g(t)}{k^{s-t}}+O\left(\xi_{1}(k)+\frac{\xi_{2}(k)}{k^{s-t}}\right)\right) .
\end{aligned}
$$

Осталось заметить, что

$$
\sum_{k \in \mathbb{N}} \frac{\mu(k)}{k^{s-t}}=\frac{1}{\zeta(s-t)}
$$

Лемма доказана.

1.4. Вспомогательные оценки. Чтобы применить лемму 1.4 к множеству $\Theta=\Omega_{R, P}$, нам понадобятся некоторые вспомогательные результаты, которые будут использоваться для оценок $\xi_{1}(k), \xi_{2}(k)$.

Пусть $\mathrm{TM}_{s}(\mathbb{Z})$ - множество целочисленных невырожденных нижнетреугольных матриц $T=\left(\left(t_{i j}\right)\right)$ размера $s \times s$ таких, что

$$
\begin{gathered}
t_{i j}=0 \quad \text { при } \quad j>i, \\
0 \leqslant t_{i j}<t_{i i} \quad \text { при } \quad j<i, \quad i=\overline{2, s}, \\
t_{11}>0 .
\end{gathered}
$$

Лемма 1.5. Любая иелочисленная матрица $M \in \mathrm{GL}_{s}(\mathbb{R})$ единственным образом представима в виде $M=T S$, где $T \in \mathrm{TM}_{s}(\mathbb{Z}), S \in \mathrm{SL}_{s}(\mathbb{Z})$.

ДокАзАТЕЛЬство существования матриц $T$ и $S$ см., например, в [8, гл. 1], единственность легко проверяется от противного.

Лемма 1.6. Пусть $M-$ целочисленная матрица размера $s \times s$. Тогда $d_{t}(M)=d_{t}(M S)$ для всех $S \in \mathrm{SL}_{s}(\mathbb{Z}), t \in\{1, \ldots, s-1\}$. 
ДоказАТЕЛьство см., например, в [9, лемма 3].

Лемма 1.7. Пусть $r \in[1,+\infty), d \in \mathbb{N}, t \in\{0,1, \ldots, s-2\}$. Тогда количество челочисленных матрии, $M \in \Omega_{R}$, удовлетворяющих условиям

$$
\begin{gathered}
d_{t+1}(M) \equiv 0(\bmod d), \\
\rho_{1}(M) \leqslant r \quad \text { либо } \quad h_{1}(M) \leqslant r,
\end{gathered}
$$

не больие чем

$$
O_{\Omega}\left(R^{s} \ln (r+1) \frac{\sigma_{-1}(d)}{d^{s-t}}\right)
$$

где $\sigma_{-1}(d)=\sum_{r \mid d} r^{-1}$.

ДокАЗАТЕЛЬСтво. Пусть

$$
T(R, d)=\left\{T \in \mathrm{TM}_{s}(\mathbb{Z}): d_{t+1}(T) \equiv 0(\bmod d), \operatorname{det} T \in[1, R]\right\},
$$

и пусть $\mathrm{M}_{s}(\mathbb{Z})$ - множество целочисленных матриц размера $s \times s$.

Согласно леммам $1.5,1.6$ любая матрица $M \in \mathrm{M}_{s}(\mathbb{Z})$ такая, что

$$
|\operatorname{det} M| \in[1, R], \quad d_{t+1}(M) \equiv 0(\bmod d),
$$

единственным образом представима в виде $M=T S$, где $T \in T(R, d), S \in$ $\mathrm{SL}_{s}(\mathbb{Z})$. Кроме того, $\Omega_{R} \cap \mathrm{M}_{s}(\mathbb{Z})=\left\{M \in \Omega \cap \mathrm{M}_{s}(\mathbb{Z}):|\operatorname{det} M| \in[1, R]\right\}$. Поэтому количество целочисленных матриц $M \in \Omega_{R}$, удовлетворяющих (1.11), (1.12), равно

$$
\sum_{T \in T(R, d)} \#\left\{M \in \Omega \cap\left(T \cdot \mathrm{SL}_{s}(\mathbb{Z})\right): \text { выполняется }(1.12)\right\} .
$$

Применяя условие iv), получаем, что искомое количество оценивается величиной

$$
O_{\Omega}(\ln (r+1) \# T(R, d)) .
$$

Осталось заметить, что \#T(R,d) $\underset{s}{\ll} R^{s} \sigma_{-1}(d) d^{-(s-t)}$ (см. $[9$, доказательство леммы 5]).

Лемма 1.8. Пусть $\varepsilon, r \in[1,+\infty)$. Тогда количество иелочисленных матрии, $M \in B\left(\Omega_{R, P}, \varepsilon\right)$ таких, что $\rho_{1}(M) \geqslant r,\left|h_{1}(M)\right| \geqslant r$, не больше чем $O_{\Omega}\left(\varepsilon R^{s} / r\right)$.

ДокАзАТЕЛьство вытекает из (1.2), (1.4), (1.5).

СлеДСтвие 1.1. Пусть $t \in\{0,1, \ldots, s-2\}, d \in \mathbb{N}, \varepsilon \in[1,+\infty)$. Тогда количество иелочисленных матрии, $M \in B\left(\Omega_{R, P}, \varepsilon\right)$, удовлетворяющих сравнению $d_{t+1}(M) \equiv 0(\bmod d)$, не больие чем

$$
O_{\Omega}\left(R^{s} \frac{\sigma_{-1}(d)(1+\ln (\varepsilon d))}{d^{s-t}}\right)
$$

ДоказАТЕЛьство. Искомое количество не больше $v_{1}+v_{2}$, где $v_{1}$ - количество целочисленных матриц $M \in \Omega_{R}$, удовлетворяющих (1.11), (1.12) при $r=\varepsilon d^{s-t}$, а $v_{2}-$ количество целочисленных матриц $M \in B\left(\Omega_{R, P}, \varepsilon\right)$ таких, что $\min \left\{\left|h_{1}(M)\right|, \rho_{1}(M)\right\} \geqslant r=\varepsilon d^{s-t}$. Осталось применить леммы $1.7,1.8$ для оценки $v_{1}$ и $v_{2}$ соответственно. 
1.5. Основные результаты. Всюду далее (до конца параграфа) предполагается, что

$$
P, R \in[1,+\infty), \quad C_{P, R}=\max \left\{1, \ln \left(P^{s-1} / R\right)\right\} .
$$

Лемма 1.9. Пусть $t \in\{0,1, \ldots, s-2\}$ и $F(t+1)$ - количество иелочисленных матрии, $M \in \Omega_{R, P}, d_{t+1}(M)=1$. Тогда

$$
F(t+1)=\left(\prod_{k=s-t}^{s} \zeta(k)\right)^{-1} F(0)+O_{\Omega}\left(R^{s}\right),
$$

где $F(0)$ - количество челочисленных матрии, $M \in \Omega_{R, P}$.

ДокАЗАТЕЛЬСтво. Используем индукцию по $t=0,1,2, \ldots, s-2$.

При $t=0$ утверждение леммы тривиально. Проведем индукционный переход от $t$ к $t+1$. Согласно лемме 1.4

$$
F(t+1)=\frac{1}{\zeta(s-t)} F(t)+O\left(\sum_{k \in \mathbb{N}}\left(\xi_{1}(k)+\frac{\xi_{2}(k)}{k^{s-t}}\right)\right),
$$

где $\xi_{1}(k)$ - количество целочисленных матриц $M \in B\left(\Omega_{R, P}, 2 k^{s-t}\right)$ таких, что $d_{t+1}(M) \equiv 0(\bmod k)$, а $\xi_{2}(k)$ - количество целочисленных матриц $M \in$ $B\left(\Omega_{R}, 2 k^{s-t}\right)$.

Используя следствие 1.1 для оценок $\xi_{1}(k)$ и $\xi_{2}(k)$ (выбираем $\varepsilon=2 k^{s-t}, d=k$ для $\xi_{1}$ и $\varepsilon=2 k^{s-t}, d=1$ для $\left.\xi_{2}\right)$, получаем

$$
\begin{gathered}
\xi_{1}(k) \underset{\Omega}{\ll} R^{s} \frac{\sigma_{-1}(k)(\ln k+1)}{k^{s-t}}, \quad \xi_{2}(k) \underset{\Omega}{\ll} R^{s} \sigma_{-1}(k)(\ln k+1), \\
\sum_{k \in \mathbb{N}}\left(\xi_{1}(k)+\frac{\xi_{2}(k)}{k^{s-t}}\right) \underset{\Omega}{\ll} R^{s} \sum_{k \in \mathbb{N}} \frac{\sigma_{-1}(k)(1+\ln k)}{k^{s-t}} \ll R^{s} .
\end{gathered}
$$

Осталось применить (1.13) и предположение индукции.

СлеДСТвиЕ 1.2. Количество иелочисленных матрии, $M \in \Omega_{R, P}$, удовлетворяющих условию $d_{s-1}(M)=1$, равно

$$
\frac{R^{s}}{s}\left(\frac{\mu(\Omega)}{\zeta(2) \cdots \zeta(s)} \ln P+O_{\Omega}\left(C_{P, R}\right)\right) .
$$

ДокАЗАТЕЛЬство вытекает из лемм 1.9, 1.3.

Лемма 1.10. Количество челочисленных матрии, $M \in \Omega_{R}$ таких, что $\rho_{1}(M) \leqslant P<\rho_{s}(M)$, не больше чем $O_{s, C}\left(R^{s}\right)$.

ДОКАЗАТЕЛЬСТВо. Используя условия іi), получаем, что искомое число не превосходит $O(S(R, P, C, s))$, где

$$
S(R, P, C, s)=\sum\left(h^{s-1} \prod_{i=1}^{s} \rho_{i}^{s-2}\right)
$$


- сумма по всем целым $(\rho, h)=\left(\rho_{1}, \ldots, \rho_{s}, h\right) \in \mathbb{Z}_{+}^{s+1}$ таким, что

$$
\begin{gathered}
\rho \in \Upsilon=\left\{\rho \in \mathbb{Z}_{+}^{s}: \rho_{i} \ll \rho_{i+1}, i=\overline{1, s-1} ; \rho_{1} \ll P \ll \rho_{s}\right\}, \\
h \prod_{i=2}^{s} \rho_{i} \ll R .
\end{gathered}
$$

Здесь и далее в этом доказательстве постоянные в оценках $O(\ldots)$ и « зависят только от $s$ и $C$. Суммируя в (1.15) по $h \ll R /\left(\rho_{2} \cdots \rho_{s}\right)$, получаем

$$
S(R, P, C, s) \ll R^{s} \sum_{\rho \in \Upsilon} \frac{\rho_{1}^{s-2}}{\rho_{2}^{2} \cdots \rho_{s}^{2}} .
$$

Множество $\Upsilon$ разобьем на части $\Upsilon_{1}, \ldots, \Upsilon_{s-1}$ так, что

$$
\forall \rho \in \Upsilon_{l} \quad \rho_{l} \ll P \ll \rho_{l+1} .
$$

Возьмем любое $l \in\{1, \ldots, s-1\}$. Суммируя в (1.16) сначала по $\rho_{i} \gg P, i=$ $\overline{l+1, s}$, а потом по $\rho_{1} \ll \rho_{2}, \rho_{2} \ll \rho_{3}, \ldots, \rho_{l-1} \ll \rho_{l}, \rho_{l} \ll P$, получаем

$$
\begin{aligned}
R^{s} \sum_{\rho \in \Upsilon_{l}} \frac{\rho_{1}^{s-2}}{\rho_{2}^{2} \cdots \rho_{s}^{2}} & \ll \frac{R^{s}}{P^{s-l}} \sum \frac{\rho_{1}^{s-2}}{\rho_{2}^{2} \rho_{3}^{2} \cdots \rho_{l}^{2}} \\
& \ll \frac{R^{s}}{P^{s-l}} \sum \frac{\rho_{2}^{s-3}}{\rho_{3}^{2} \cdots \rho_{l}^{2}} \ll \cdots \ll \frac{R^{s}}{P^{s-l}} \sum_{1 \leqslant \rho_{l} \ll P} \rho_{l}^{s-l-1} \ll R^{s} .
\end{aligned}
$$

В силу произвольности $l$ доказали, что $S(R, P, C, s) \ll R^{s}$.

СлеДСтвиЕ 1.3. Количество иелочисленных матрии, $M \in \Omega_{R}$, удовлетворяющих условиям

$$
d_{s-1}(M)=1, \quad \rho_{1}(M) \leqslant P
$$

равно (1.14).

ДокАЗАТЕЛЬСТво вытекает из леммы 1.10 и следствия 1.2.

СлеДСтвиЕ 1.4. Количество иелочисленных матрии, $M \in \Omega_{R}$, удовлетворяющих условиям

$$
d_{s-1}(M)=1, \quad \rho_{1}(M) \leqslant P, \quad|\operatorname{det} M| P^{-s} \leqslant h_{1}(M),
$$

равно (1.14).

ДокАЗАТЕЛьство. Пусть $M$ - целочисленная матрица из $\Omega_{R}$ такая, что $\rho_{1}(M) \leqslant P$ и $h_{1}(M)<|\operatorname{det} M| P^{-s}$. Применяя условия ii), получаем

$$
|\operatorname{det} M| \underset{C, s}{\ll} h_{1}(M) \prod_{j=2}^{s} \rho_{j}(M) \underset{C, s}{\ll}\left(|\operatorname{det} M| P^{-s}\right)\left(\rho_{s}(M)\right)^{s-1} .
$$

Следовательно, $\rho_{s}(M) \underset{C, s}{\gg} P$. Согласно лемме 1.10 таких матриц не больше чем $O_{C, s}\left(R^{s}\right)$. Используя следствие 1.3, приходим к формуле (1.14). 


\section{§ 2. Цилиндрические минимумы}

Так же, как и в предыдущем параграфе, считаем, что $s=n+1$.

Пусть Г - полная решетка (в дальнейшем слово “полная" не используем) в $\mathbb{R}^{s}$, т. е. это множество вида

$$
\Gamma=\left\{n_{1} a^{(1)}+\cdots+n_{s} a^{(s)}: n_{i} \in \mathbb{Z}, i=\overline{1, s}\right\},
$$

где $a^{(1)}, \ldots, a^{(s)}$ - некоторые линейно независимые векторы из $\mathbb{R}^{s}$ (базис $Г$ ). Матрицу со столбцами $\left(a^{(1)}\right)^{T}, \ldots,\left(a^{(s)}\right)^{T}$ будем называть базисной. Модуль определителя базисной матрицы называется определителем решетки и обозначается $\operatorname{det} \Gamma$.

Ненулевой узел $а$ решетки Г будем называть иилиндрическим $f$-минимумом, если не существует узла $\gamma \in \Gamma \backslash\{0\}$ такого, что

$$
f\left(\gamma_{1}, \ldots, \gamma_{s-1}\right) \leqslant f\left(a_{1}, \ldots, a_{s-1}\right), \quad\left|\gamma_{s}\right| \leqslant\left|a_{s}\right|,
$$

причем хотя бы одно из неравенств является строгим.

Цилиндрические минимумы (при $s=3, f(x)=\sqrt{x_{1}^{2}+x_{2}^{2}}$ ) впервые появились в работах Г.Ф. Вороного (см. [10], [11]) в связи с методами построения единиц в кубических числовых полях отрицательного дискриминанта. Они также естественным образом возникают в теории многомерных приближений.

В этом параграфе мы приведем некоторые результаты из статьи [7], в которой рассмотрен вопрос о среднем количестве цилиндрических минимумов целочисленных решеток. Отметим, что в [7] предполагалось, что функция $f$ является нормой. Однако все результаты из [7], которые приводятся в настоящей работе без доказательства, остаются в силе, если $f$ является только лучевой функцией (неравенство треугольника в [7] использовалось только при обосновании оценки (2.5)).

2.1. Приведенные базисы. Базис $e^{(1)}, \ldots, e^{(m)}$ решетки $\Lambda \subset \mathbb{R}^{m}$ называется приведенным (по Минковскому), если выполняются следующие условия:

1) $\left|e^{(1)}\right|=\min \{|\gamma|: \gamma \in \Lambda \backslash\{0\}\}$

2) если $j \geqslant 2$, то $\left|e^{(j)}\right|=\min |\eta|$, где минимум берется по всем узлам $\eta \in \Lambda$ таким, что система $e^{(1)}, \ldots, e^{(j-1)}, \eta$ дополняется до базиса решетки $\Lambda$.

Известно, что (см., например, [8, гл. II, §2]) базис $\left\{e^{(i)}\right\}_{i=1}^{m}$ является приведенным тогда и только тогда, когда для любого $j \in\{1, \ldots, m\}$

$$
\left|e^{(j)}\right| \leqslant\left|\sum_{i=1}^{m} k_{i} e^{(i)}\right|
$$

при всех $\left(k_{1}, \ldots, k_{m}\right) \in \mathbb{Z}^{m}$ таких, что НОД $\left(k_{j}, k_{j+1}, \ldots, k_{m}\right)=1$.

Введем следующее отношение упорядоченности на $\mathbb{R}^{m}$ (лексикографическое). Пусть $x, y \in \mathbb{R}^{m}$. Будем записывать $x \prec y$, если существует такой номер $l \in\{1, \ldots, m\}$, что

$$
x_{i}=y_{i}, \quad i=\overline{1, l-1} ; \quad x_{l}<y_{l} .
$$


Пусть $\left\{e^{(i)}\right\}_{i=1}^{m}$ и $\left\{f^{(i)}\right\}_{i=1}^{m}$ - два набора векторов из $\mathbb{R}^{m}$. Записываем $\left\{e^{(i)}\right\}_{i=1}^{m} \prec$ $\left\{f^{(i)}\right\}_{i=1}^{m}$, если существует такой номер $l \in\{1, \ldots, m\}$, что

$$
e^{(i)}=f^{(i)}, \quad i=\overline{1, l-1} ; \quad e^{(l)} \prec f^{(l)} .
$$

Приведенный базис $\left\{e^{(i)}\right\}_{i=1}^{m}$ решетки $\Lambda$ будем называть вполне приведенным, если $\left\{e^{(i)}\right\}_{i=1}^{m} \prec\left\{f^{(i)}\right\}_{i=1}^{m}$ для любого другого приведенного базиса $\left\{f^{(i)}\right\}_{i=1}^{m}$ решетки $\Lambda$.

Любая решетка имеет единственный вполне приведенный базис.

Пусть

$$
\widetilde{\mathfrak{R}}_{m} \subset \underbrace{\mathbb{R}^{m} \times \cdots \times \mathbb{R}^{m}}_{m}
$$

- множество, составленное из всех приведенных базисов $m$-мерных решеток. Согласно известным свойствам приведенных квадратичных форм (см., например, [13, гл. 12, теорема 1.3]) существует такое конечное множество (зависящее только от размерности $m$ ) условий вида (2.2), что если базис $\left\{e^{(i)}\right\}_{i=1}^{m}$ удовлетворяет условиям этого множества, то $\left\{e^{(i)}\right\}_{i=1}^{m}-$ приведенный базис. В частности, это означает, что граница множества $\widetilde{\Re}_{m}$ состоит из фиксированного числа поверхностей класса $C^{\infty}$.

Рассмотрим теперь множество $\mathfrak{R}_{m} \subset \widetilde{\mathfrak{R}}_{m}$, составленное из всех вполне приведенных базисов $m$-мерных решеток. Если $\left\{e^{(i)}\right\}_{i=1}^{m}-$ приведенный базис $\Lambda$, то элементы матрицы перехода от $\left\{e^{(i)}\right\}_{i=1}^{m}$ к любому другому приведенному базису решетки $\Lambda$ ограничены некоторой константой, зависящей только от $m$ (см., например, [13, гл. 12, теорема 1.2]). Поэтому граница множества $\mathfrak{R}_{m}$ также состоит из фиксированного числа поверхностей класса $C^{\infty}$.

2.2. Базисы Вороного и их свойства. Рассмотрим метод дополнения примитивного узла решетки до базиса, предложенный Г. Ф. Вороным. Используем определения из $[12$, гл. $5, \S 60])$.

Пусть $\Gamma$ - решетка из $\mathbb{R}^{s}$. Возьмем любой примитивный узел $a=\left(a_{1}, \ldots\right.$ $\left.\ldots, a_{s}\right) \in \Gamma$, где $a_{s}>0$. Все точки решетки лежат на прямых, параллельных $a$ (nараллеля $)$. Эти прямые пересекают плоскость $\pi_{s}=\left\{x \in \mathbb{R}^{s}: x_{s}=0\right\}$ в узлах некоторой решетки $\Lambda$, которую можно рассматривать как $(s-1)$-мерную полную решетку, если вычеркнуть последние координаты узлов (которые равны нулю). Узел решетки $\Lambda$ будем называть основанием параллели. Отрезок параллели от основания до первой, лежащей не ниже плоскости $\pi_{s}$, точки решетки Г будем называть гвоздиком, первую точку решетки - шапочкой гвоздика, а основание параллели - основанием гвоздика.

Отметим, что гвоздик может иметь нулевую длину (если его основание является узлом Г). Шапочка $\gamma$ и основание $\eta$ гвоздика связаны между собой соотношением

$$
\eta=\gamma-a \frac{\gamma_{s}}{a_{s}}
$$

Узел $\gamma \in \Gamma$ является шапочкой некоторого гвоздика, если и только если $0 \leqslant \gamma_{s}<a_{s}$. Поэтому для любого гвоздика с шапочкой $\gamma$ и основанием $\eta$

$$
\rho(\gamma)-\rho(a) \leqslant|\eta| \leqslant \rho(\gamma)+\rho(a) .
$$


Пусть $\left\{b^{(j)}\right\}_{j=2}^{s}-$ вполне приведенный базис $\Lambda$. Обозначим $a^{(1)}=a$, и пусть $a^{(j)}$ - шапочка гвоздика с основанием в точке $b^{(j)}, j \geqslant 2$. Тогда $\left\{a^{(j)}\right\}_{j=1}^{s}$ будет базисом Г. Назовем его базисом Вороного.

Будем говорить, что ненулевой узел $\gamma \in \Gamma$ нарушает минимальность узла $a$, если выполняются неравенства (2.1), причем хотя бы одно из них является строгим.

Лемма 2.1. Пусть шапочки гвоздиков с основаниями в точках $\pm b^{(2)}$ не нарушают минимальность узла $a^{(1)}$. Положим $\rho_{j}=\rho\left(a^{(j)}\right), j=\overline{1, s}$. Тогда

$$
\begin{gathered}
\rho_{1} \underset{f}{\ll}\left|b^{(2)}\right|, \\
a_{s} \prod_{j=2}^{s} \rho_{j} \underset{f}{\ll} \operatorname{det} \Gamma .
\end{gathered}
$$

Если, дополнительно, узль $a^{(3)}, \ldots, a^{(s)}$ не нарушают минимальность узла $a^{(1)}$, mo

$$
\begin{gathered}
\rho_{j} \underset{f}{\overparen{f}}\left|b^{(j)}\right|, \quad j=\overline{2, s}, \\
\rho_{j} \underset{f}{\ll} \rho_{j+1}, \quad j=\overline{1, s-1 .}
\end{gathered}
$$

ДокАЗАТЕльство. Для удобства записи будем считать, что функция $f$ определена на всем $\mathbb{R}^{s}$ по правилу

$$
f(x)=f\left(x_{1}, \ldots, x_{s-1}\right) \quad \text { при } \quad x=\left(x_{1}, \ldots, x_{s-1}, x_{s}\right) \in \mathbb{R}^{s} .
$$

Поскольку $f$ - непрерывная и лучевая функция, то

$$
\forall x \in \mathbb{R}^{s} \quad f(x) \asymp \varlimsup_{f} \rho(x) .
$$

Так как функция $f$ непрерывна и кусочно дифференцируема, то она удовлетворяет условию Липшица, т.е. существует такая постоянная $l>0$, что для любых $x, y \in \mathbb{R}^{s}$

$$
|f(x)-f(y)| \leqslant l|x-y| .
$$

Перепишем последнюю оценку в следующем виде:

$$
f(x+y) \leqslant f(y)+l|x| .
$$

Если шапочка $\gamma$ некоторого гвоздика не нарушает минимальность $a$, то $f(a)<f(\gamma)$ и согласно $(2.9)$

$$
\rho_{1} \underset{f}{\ll} \rho(\gamma)
$$

Докажем (2.5). Пусть $\widetilde{a}$ - шапочка гвоздика с основанием в точке $\left(-b^{(2)}\right)$. Согласно (2.3) узел $a^{(2)}$ и шапочку $\widetilde{a}$ можно представить в виде

$$
a^{(2)}=b^{(2)}+t a, \quad \widetilde{a}=-b^{(2)}+(1-t) a
$$


с некоторым $t \in[0,1)$. Поскольку $\widetilde{a}$ и $a^{(2)}$ не могут нарушать минимальность $a$, то, учитывая (2.10), получаем

$$
\begin{gathered}
f(a)<f\left(a^{(2)}\right)=f\left(b^{(2)}+t a\right) \leqslant t f(a)+l\left|b^{(2)}\right|, \\
f(a)<f(\widetilde{a})=f\left(-b^{(2)}+(1-t) a\right) \leqslant(1-t) f(a)+l\left|b^{(2)}\right| .
\end{gathered}
$$

Следовательно, $f(a)<2 l\left|b^{(2)}\right|$, и, используя (2.9), приходим к (2.5).

Неравенство (2.6) доказано в [7, лемма 1]. Выведем (2.7). Возьмем любое $j \in\{2, \ldots, s\}$. Согласно (2.4) имеем

$$
\left|b^{(j)}\right| \leqslant \rho_{j}+\rho_{1}, \quad \rho_{j} \leqslant\left|b^{(j)}\right|+\rho_{1} .
$$

Поскольку $a^{(j)}$ не нарушает минимальность $a$, то

$$
\rho_{1} \underset{f}{\ll} \rho_{j} .
$$

Используя (2.5) и определение приведенного базиса, получаем

$$
\rho_{1} \underset{f}{\ll}\left|b^{(2)}\right| \leqslant\left|b^{(j)}\right| .
$$

Из этих соотношений следуют оценки (2.7).

Неравенства (2.8) вытекают из (2.7) и оценок $\left|b^{(j)}\right| \leqslant\left|b^{(j+1)}\right|$.

Лемма 2.2. Существует такое конечное множество $\mathfrak{C}_{f} \subset \mathbb{Z}^{s}$, зависящее только от $f$, что если $\left\{a^{(j)}\right\}_{j=1}^{s}$ - базис Вороного решетки Г и узлы вида

$$
\gamma=\sum_{i=1}^{s} k_{i} a^{(i)}, \quad\left(k_{1}, \ldots, k_{s}\right) \in \mathfrak{C}_{f}
$$

не нарушают минимальность $a^{(1)}$, то $a^{(1)}$ является иилиндрическим $f$-минимумом решетки Г.

ДокАЗАТЕЛЬСтво см. в [7, следствие 1].

Рассмотрим базис Вороного $\left\{a^{(j)}\right\}_{j=1}^{s}$ решетки $\Gamma$ из $\mathbb{R}^{s}$, удовлетворяющий следующему дополнительному условию: $a^{(1)}$ - цилиндрический $f$-минимум $\Gamma$. Пусть $A=\left(\left(a_{i}^{(j)}\right)\right)$ - соответствующая базисная матрица $\left(\right.$ со столбцами $\left(a^{(1)}\right)^{T}, \ldots$ $\left.\ldots,\left(a^{(s)}\right)^{T}\right)$. Будем называть ее матрицей Вороного.

Пусть $\Omega_{f}$ - множество, состоящее из всех матриц Вороного решеток из $\mathbb{R}^{s}$.

Любая матрица $A=\left(\left(a_{i}^{(j)}\right)\right) \in \Omega_{f}$ обладает следующими свойствами:

ј) справедливы оценки $0 \leqslant a_{s}^{(j)}<a_{s}^{(1)}, j=\overline{2, s}$;

jj) если

$$
b^{(j)}=a^{(j)}-a^{(1)} \frac{a_{s}^{(j)}}{a_{s}^{(1)}}, \quad j=\overline{2, s},
$$

- основания гвоздиков с шапочками в точках $a^{(j)}$, то $\left(b^{(2)}, \ldots, b^{(s)}\right)$ содержится во множестве вполне приведенных базисов $\mathfrak{R}_{s-1}$;

jjj) узлы (2.11) не нарушают минимальность узла $a^{(1)}$. 
Верно и обратное: если матрица $A \in \mathrm{GL}_{s}(\mathbb{R})\left(\right.$ со столбцами $\left(a^{(1)}\right)^{T}, \ldots$ $\left.\ldots,\left(a^{(s)}\right)^{T}\right)$ удовлетворяет j)-jjj), то $A \in \Omega_{f}$.

Множество $\Omega=\Omega_{f}$ удовлетворяет условиям i)-iii) из $\S 1$. Выполнение условия i) вытекает из определения матрицы Вороного, условия іi) - из j) и соотношений (2.6), (2.8). Условие iii) следует из j)-jjj).

2.3. О множестве $\Omega_{f}$ при $s=3$. Можно уточнить условия јjј) в следующем частном случае:

$$
s=3, \quad f(x)=\sqrt{x_{1}^{2}+x_{2}^{2}} .
$$

Пусть $a=\left(a_{1}, a_{2}, a_{3}\right)$ - примитивный узел трехмерной решетки $\Gamma$ и $a_{3}>0$. Пусть $l_{a}-$ луч, выходящий из начала координат и проходящий через точку $\left(a_{1}, a_{2}, 0\right)$. На двумерной решетке оснований гвоздиков $\Lambda$ определим шестиугольник Зеллинга как шестиугольник, составленный из шести остроугольных (или прямоугольных) примитивных треугольников решетки $\Lambda$, сходящихся в начале координат. Определим основной треугольник Зеллинга как тот из шести треугольников, через который проходит луч $l_{a}$.

Рассмотрим шапочки семи гвоздиков с основаниями в вершинах шестиугольника Зеллинга и в точке $(\beta+\gamma)$, где $\beta$ и $\gamma$ - вершины основного треугольника Зеллинга, отличные от нуля. Будем называть их шапочками Вороного.

Теорема Вороного. Пусть выполняются условия (2.12). Если шапочки Вороного не нарушают минимальность узла а, то а является иилиндрическим $f$-минимумом.

Данная теорема была доказана Г. Ф. Вороным [10], [11] (переработанное доказательство см. в $[12$, гл. $5, \S 60])$ в случае, когда решетка Г удовлетворяет условию (решетка общего положения)

$$
\rho(\gamma) \neq \rho(\eta), \quad\left|\gamma_{s}\right| \neq\left|\eta_{s}\right|, \quad \gamma, \eta \in \Gamma, \quad \eta \neq \gamma
$$

Доказательство этой теоремы для произвольных решеток, в том числе и целочисленных, см. в [14].

Таким образом, в случае (2.12) условие jjj) можно заменить на следующее:

$\mathrm{jjj}^{\prime}$ ) шапочки Вороного не нарушают минимальность $a$.

ЗАмЕчАниЕ 2.1. Если (2.13) не выполняется, то основной треугольник Зеллинга (и даже шестиугольник Зеллинга) может определяться неоднозначно. Однако утверждение теоремы Вороного остается в силе независимо от выбора этого треугольника (и шестиугольника). Отметим, что в этом случае число рассматриваемых шапочек Вороного можно уменьшить (см. [14]).

2.4. Среднее количество цилиндрических минимумов. Определим отображение $\Phi$, которое каждой матрице $X \in \mathrm{GL}_{s}(\mathbb{R})$ ставит в соответствие пару $(\Gamma(X), a(X))$, где:

1) $\Gamma(X)$ - решетка с базисной матрицей $X$ (т. е. столбцы $X$ образуют базис $\Gamma(X))$;

2) $a(X)=\left(x_{11}, \ldots, x_{s 1}\right)$ - узел $\Gamma(X)$ (первый столбец $\left.X\right)$. 
Пусть $\mathfrak{M}_{f}(\Gamma)$ - множество цилиндрических $f$-минимумов решетки $Г$ и $\mathfrak{M}_{f}^{+}(\Gamma)$ - множество минимумов $a \in \mathfrak{M}_{f}(\Gamma)$, удовлетворяющих условию $a_{s}>0$.

Определим множество $\mathcal{U}_{f}$, состоящее из пар $(\Gamma, a)$, где $\Gamma$ - решетка в $\mathbb{R}^{s}$ и $a \in \mathfrak{M}_{f}^{+}(\Gamma)$.

ЗАмечАниЕ 2.2. Отображение $\Phi$ взаимно однозначно переводит $\Omega_{f}$ на все множество $\mathcal{U}_{f}$ (это вытекает из определений). Кроме того, если $M(\Gamma)$ - множество базисных матриц решетки $Г$, то множество цилиндрических минимумов с $a_{s}>0$ определяется формулой

$$
\mathfrak{M}_{f}^{+}(\Gamma)=\left\{a(M): M \in \Omega_{f} \cap M(\Gamma)\right\},
$$

т. е. оно состоит из первых столбцов базисных матриц Вороного решетки Г. Последнее соотношение можно записать следующим образом:

$$
\forall X \in M(\Gamma) \quad \mathfrak{M}_{f}^{+}(\Gamma)=\left\{a(Y): Y \in \Omega_{f} \cap\left(X \cdot \mathrm{SL}_{s}(\mathbb{Z})\right)\right\} .
$$

Используя замечание 2.2, можно сводить вычисление усредненного по некоторому множеству решеток числа цилиндрических минимумов к нахождению количества матриц из некоторого подмножества $\Omega_{f}$. Так, в [7] была доказана асимптотическая формула

$$
\frac{1}{\# \mathcal{L}_{s}(\mathbb{Z} ;[1, R])} \sum_{\Gamma \in \mathcal{L}_{s}(\mathbb{Z} ;[1, R])} \# \mathfrak{M}_{f}(\Gamma)=\frac{\mathcal{C}_{f}}{s-1} \ln R+O_{f}(1),
$$

где $\mathcal{L}_{s}(\mathbb{Z} ;[1, R])$ - множество $s$-мерных целочисленных решеток $Г$ таких, что $\operatorname{det} \Gamma \in[1, R]$,

$$
\mathcal{C}_{f}=2 \cdot \frac{\mu\left(\Omega_{f}\right)}{\zeta(2) \cdots \zeta(s)}
$$

В настоящей статье мы ограничимся доказательством тех результатов, которые потребуются при исследовании наилучших приближений линейных форм. Для вычисления мощности подмножеств $\Omega_{f}$ будем использовать результаты из $\S 1$. Как уже отмечалось, множество $\Omega=\Omega_{f}$ удовлетворяет условиям i)-iii) из §1. Докажем выполнение iv).

Возьмем любую решетку $Г$ из $\mathbb{R}^{s}$ и положительные вещественные $r_{2} \geqslant r_{1}$, $h_{2} \geqslant h_{1}$. Согласно [2, теорема 1.1] справедливы оценки

$$
\begin{aligned}
& \#\left\{a \in \mathfrak{M}_{f}(\Gamma): r_{1} \leqslant \rho(a) \leqslant r_{2}\right\} \underset{f}{\ll} \ln \left(\frac{r_{2}}{r_{1}}+1\right), \\
& \#\left\{a \in \mathfrak{M}_{f}(\Gamma): h_{1} \leqslant\left|a_{s}\right| \leqslant h_{2}\right\} \underset{f}{\ll} \ln \left(\frac{h_{2}}{h_{1}}+1\right) .
\end{aligned}
$$

Лемма 2.3. Множество $\Omega=\Omega_{f}$ удовлетворяет условию іv) из $\S 1$.

ДоказАтельство. Пусть $r \in[1,+\infty)$ и $Q$ - целочисленная матрица из $\Omega_{f}$. Согласно (2.14) количество матриц $M \in \Omega_{f}\left(Q \cdot \mathrm{SL}_{s}(\mathbb{Z})\right)$, удовлетворяющих условию $\rho_{1}(M) \leqslant r$ либо $h_{1}(M) \leqslant r$, не больше чем число минимумов $a \in \mathfrak{M}_{f}(\Gamma(Q))$, для которых $\rho(a) \leqslant r$ либо $\left|a_{s}\right| \leqslant r$. Используя $(2.16),(2.17)$, мы видим, что количество таких минимумов не превосходит $O_{f}(\ln r+1)$. Лемма доказана. 
Для любых $\mathbf{P}=\left(P_{1}, \ldots, P_{s-1}\right) \in \mathbb{Z}_{+}^{s-1}$ и $N \in \mathbb{N}$ определим решетку $\Gamma(\mathbf{P}, N)$ с базисной матрицей

$$
T=\left(\begin{array}{ccccc}
1 & 0 & \ldots & 0 & 0 \\
0 & 1 & \ldots & 0 & 0 \\
\ldots & \ldots & \ldots & \ldots & \ldots \\
0 & 0 & \ldots & 1 & 0 \\
P_{1} & P_{2} & \ldots & P_{s-1} & N
\end{array}\right)
$$

Пусть $\mathcal{L}_{s}(R)$ - множество, состоящее из решеток $\Gamma(\mathbf{P}, N)$ с целыми $P_{i}, N$, удовлетворяющими неравенствам $0 \leqslant P_{i}<N \leqslant R, i=\overline{1, s-1}$. Отметим, что

$$
\# \mathcal{L}_{s}(R)=\frac{R^{s}}{s}+O_{s}\left(R^{s-1}\right) .
$$

Выясним, какие матрицы порождают решетки из $\mathcal{L}_{s}(R)$.

Лемма 2.4. Пусть $M$ - целочисленная матрица из $\mathrm{GL}_{s}(\mathbb{R})$. Пусть $\Gamma=$ $\Gamma(M)$ - решетка с базисной матрищей $M$. Тогда $\Gamma \in \mathcal{L}_{s}(R)$, если и только если $|\operatorname{det} M| \in[1, R], d_{s-1}(M)=1$.

Доказательство. Пусть $\Gamma \in \mathcal{L}_{s}(R)$. Тогда $\operatorname{det} \Gamma \in[1, R]$, и поэтому $|\operatorname{det} M| \in[1, R]$. Кроме того, существует матрица $S \in \mathrm{SL}_{s}(\mathbb{Z})$ такая, что $M=T S$, где $T$ - матрица вида (2.18). Поскольку $d_{s-1}(T)=1$, согласно лемме 1.6 имеем $d_{s-1}(M)=1$.

Пусть $|\operatorname{det} M| \in[1, R], d_{s-1}(M)=1$. Согласно лемме 1.5 матрицу $M$ можно представить в виде $M=T S$, где $S \in \mathrm{SL}_{s}(\mathbb{Z})$, а $T \in \mathrm{TM}_{s}(\mathbb{Z})$ - еще одна базисная матрица $\Gamma$. Поскольку $d_{s-1}(M)=1$, по лемме 1.6 имеем $d_{s-1}(T)=1$. Значит, $t_{i i}=1, i=\overline{1, s-1}$, и, следовательно, матрица $T$ имеет вид (2.18). Так как $|\operatorname{det} M| \in[1, R]$, то $N \leqslant R$. Лемма доказана.

Для любого $\alpha=\left(\alpha_{1}, \ldots, \alpha_{s-1}\right) \in[0,1)^{s-1}$ определим решетку $\Gamma_{\alpha}$ с базисной матрицей

$$
T_{\alpha}=\left(\begin{array}{ccccc}
1 & 0 & \ldots & 0 & 0 \\
0 & 1 & \ldots & 0 & 0 \\
\ldots & \ldots & \ldots & \ldots & \ldots \\
0 & 0 & \ldots & 1 & 0 \\
\alpha_{1} & \alpha_{2} & \ldots & \alpha_{s-1} & 1
\end{array}\right)
$$

т. е. $\Gamma_{\alpha}$ состоит из узлов вида

$$
\gamma=\left(k_{1}, k_{2}, \ldots, k_{s-1}, k_{1} \alpha_{1}+\cdots+k_{s-1} \alpha_{s-1}-k_{s}\right), \quad k_{i} \in \mathbb{Z} .
$$

Рассмотрим функции $\varphi, \psi:[0,1)^{s-1} \rightarrow \mathbb{Z}_{+}$, действующие по формулам

$$
\begin{gathered}
\varphi(\alpha)=\#\left\{a \in \mathfrak{M}_{f}^{+}\left(\Gamma_{\alpha}\right): f\left(a_{1}, \ldots, a_{s-1}\right) \leqslant P\right\} \\
\psi(\alpha)=\#\left\{a \in \mathfrak{M}_{f}^{+}\left(\Gamma_{\alpha}\right): f\left(a_{1}, \ldots, a_{s-1}\right) \leqslant P, P^{-s} \leqslant a_{s}\right\} .
\end{gathered}
$$

Множество векторов $\Delta_{s-1}(R)$ определено во введении. 
Лемма 2.5. Для любых $P, R \in(1,+\infty)$ имеют место асимптотические формульь

$$
\begin{aligned}
& \frac{1}{\# \Delta_{s-1}(R)} \sum_{\alpha \in \Delta_{s-1}(R)} \varphi(\alpha)=\frac{\mathcal{C}_{f}}{2} \ln P+O_{f}\left(\frac{P^{s-1}}{R}+1\right), \\
& \frac{1}{\# \Delta_{s-1}(R)} \sum_{\alpha \in \Delta_{s-1}(R)} \psi(\alpha)=\frac{\mathcal{C}_{f}}{2} \ln P+O_{f}\left(\frac{P^{s-1}}{R}+1\right),
\end{aligned}
$$

где постоянная $\mathcal{C}_{f}$ определяется формулой (2.15).

ДокАзАтельство. Докажем сначала (2.23). Пусть

$$
\alpha=\left(\frac{P_{1}}{N}, \ldots, \frac{P_{s-1}}{N}\right) \in \Delta_{s-1}(R) .
$$

Отображение

$$
\left(x_{1}, \ldots, x_{s-1}, x_{s}\right) \in \mathbb{R}^{s} \rightarrow\left(x_{1}, \ldots, x_{s-1}, N x_{s}\right)
$$

взаимно однозначно переводит $\Gamma_{\alpha}$ на решетку $\Gamma=\Gamma(\mathbf{P}, N) \in \mathcal{L}_{s}(R)$, при этом минимумы $\Gamma_{\alpha}$ переходят в минимумы Г. Поэтому $\varphi(\alpha)=\varphi(\Gamma)$, где $\varphi(\Gamma)-$ количество минимумов $a \in \mathfrak{M}_{f}^{+}(\Gamma)$, удовлетворяющих условию $f\left(a_{1}, \ldots, a_{s-1}\right) \leqslant P$. Значит, достаточно доказать, что

$$
\frac{1}{\# \mathcal{L}_{s}(R)} \sum_{\Gamma \in \mathcal{L}_{s}(R)} \varphi(\Gamma)=\frac{\mathcal{C}_{f}}{2} \ln P+O_{f}\left(\frac{P^{s-1}}{R}+1\right) .
$$

Возьмем любую решетку $\Gamma \in \mathcal{L}_{s}(R)$. Пусть $\widetilde{\varphi}(\Gamma)$ - количество минимумов $a \in \mathfrak{M}_{f}^{+}(\Gamma)$ таких, что $\rho(a) \leqslant P$. Если $a \in \mathfrak{M}_{f}(\Gamma)$, причем $\rho(a) \leqslant P \leqslant f(a)$ либо $f(a) \leqslant P \leqslant \rho(a)$, то согласно $(2.9)$ имеем $\rho(a) \underset{f}{\asymp} P$. Используя оценку $(2.16)$, получаем, что таких минимумов не больше чем $O_{f}(1)$. Следовательно, $\varphi(\Gamma)=\widetilde{\varphi}(\Gamma)+O_{f}(1)$, и

$$
\sum_{\Gamma \in \mathcal{L}_{s}(R)} \varphi(\Gamma)=\sum_{\Gamma \in \mathcal{L}_{s}(R)} \widetilde{\varphi}(\Gamma)+O_{f}\left(\# \mathcal{L}_{s}(R)\right) .
$$

Пусть $\mathcal{U}_{f}^{*}=\mathcal{U}_{f}^{*}(P, R)$ - множество пар $(\Gamma, a) \in \mathcal{U}_{f}$, где $\Gamma \in \mathcal{L}_{s}(R)$ и $a-$ минимум из $\mathfrak{M}_{f}^{+}(\Gamma)$ такой, что $\rho(a) \leqslant P$. Тогда

$$
\sum_{\Gamma \in \mathcal{L}_{s}(R)} \tilde{\varphi}(\Gamma)=\# \mathcal{U}_{f}^{*}
$$

Определим множество $\Omega_{f}^{*}=\Omega_{f}^{*}(P, R)$, составленное из всех матриц Вороного для пар $(\Gamma, a)$ из $\mathcal{U}_{f}^{*}$, т. е. $\Phi\left(\Omega_{f}^{*}\right)=\mathcal{U}_{f}^{*}$. Поскольку отображение $\Phi$ взаимно однозначное, то $\# \mathcal{U}_{f}^{*}=\# \Omega_{f}^{*}$. Используя определения и лемму 2.4 , получаем, что множество $\Omega_{f}^{*}$ состоит из целочисленных матриц $M \in \Omega_{f}$ таких, что

$$
\rho_{1}(M) \leqslant P, \quad|\operatorname{det} M| \in[1, R], \quad d_{s-1}(M)=1 .
$$


Множество $\Omega=\Omega_{f}$ удовлетворяет условиям i)-iv) из $\S 1$. Поэтому согласно следствию 1.3 имеем

$$
\sum_{\Gamma \in \mathcal{L}_{s}(R)} \widetilde{\varphi}(\Gamma)=\# \mathcal{U}_{f}^{*}=\# \Omega_{f}^{*}=\frac{R^{s}}{s}\left(\frac{\mu\left(\Omega_{f}\right)}{\zeta(2) \cdots \zeta(s)} \ln P+O_{f}\left(1+\frac{P^{s-1}}{R}\right)\right) .
$$

Подставляя последнее соотношение в (2.26) и учитывая (2.19), приходим к соотношению (2.25). Оценка (2.23) доказана.

Для доказательства (2.24) достаточно повторить рассуждения, использованные при обосновании (2.23). В итоге получаем

$$
\sum_{\alpha \in \Delta_{s-1}(R)} \psi(\alpha)=\# \Omega_{f}^{* *}+O_{f}\left(\# \mathcal{L}_{s}(R)\right),
$$

где $\Omega_{f}^{* *}$ состоит из целочисленных матриц $M \in \Omega_{f}$ таких, что

$$
\rho_{1}(M) \leqslant P, \quad|\operatorname{det} M| P^{-s} \leqslant h_{1}(M), \quad|\operatorname{det} M| \in[1, R], \quad d_{s-1}(M)=1 .
$$

Применяя для вычисления $\# \Omega_{f}^{* *}$ следствие 1.4 , приходим к требуемой формуле.

\section{§ 3. Среднее количество наилучших приближений}

Напомним, что размерность $s$ решеток из предыдущего параграфа и размерность $n$ рассматриваемых линейных форм связаны соотношением $s=n+1$.

Возьмем любой вектор $\alpha=\left(\alpha_{1}, \ldots, \alpha_{n}\right) \in \mathbb{R}^{n}$. Определим линейную форму

$$
L_{\alpha} x=\alpha_{1} x_{1}+\cdots+\alpha_{n} x_{n}
$$

и решетку $\Gamma_{\alpha}$ с базисной матрицей (2.20). Из определений вытекает, что множество минимумов $\mathfrak{M}_{f}\left(\Gamma_{\alpha}\right)$ состоит из векторов $\left(u_{1}, \ldots, u_{n}, L_{\alpha} u-v\right)$, где $(u, v)-$ $f$-наилучшее приближение линейной формы $L_{\alpha}$. Таким образом, существует взаимно однозначное соответствие между цилиндрическими минимумами и наилучшими приближениями линейной формы. Отсюда, в частности, вытекает, что

$$
\#\left\{a \in \mathfrak{M}_{f}\left(\Gamma_{\alpha} ; P\right): f\left(a_{1}, \ldots, a_{s-1}\right) \leqslant P\right\}=\# \mathfrak{B}_{f}(\alpha ; P) .
$$

Если $a \in \mathfrak{M}_{f}\left(\Gamma_{\alpha}\right)$, то и $(-a) \in \mathfrak{M}\left(\Gamma_{\alpha}\right)$. Поэтому

$$
\# \mathfrak{B}_{f}(\alpha ; P)=2 \varphi(\alpha)+\xi(\alpha)
$$

где $\varphi(\alpha)$ определяется $(2.21)$, а $\xi(\alpha)$ - количество минимумов $a \in \mathfrak{M}_{f}\left(\Gamma_{\alpha}\right)$ таких, что $a_{s}=0, f\left(a_{1}, \ldots, a_{s-1}\right) \leqslant P$. Очевидно, что $\xi(\alpha)=O_{f}(1)$.

Рассмотрим сначала дискретный случай.

Теорема 3.1. Для любых $P, R>1$ справедлива асимптотическая формула (0.3), в которой

$$
\mathcal{C}_{f}=2 \cdot \frac{\mu\left(\Omega_{f}\right)}{\zeta(2) \cdots \zeta(n+1)}
$$


ДокАЗАТЕЛЬство вытекает из (3.1) и (2.23).

Лемма 3.1. Пусть $\alpha \in \Delta_{n}(R)$. Тогда для любого наилучшего приближения $(u, v) \in \mathfrak{B}_{f}(\alpha)$, удовлетворяющего условию $L_{\alpha} u \neq v$, имеет место оцен$\kappa a(0.2)$.

ДокАЗАТЕЛЬство. Пусть $\theta=\left|L_{\alpha} u-v\right|$. Тогда $\theta \geqslant R^{-1}$. Согласно (2.9) и определению наилучшего приближения существует такая постоянная $C=$ $C(f)>0$, что выпуклое множество

$$
U(\gamma)=\left\{x \in \mathbb{R}^{s}: \rho(x) \leqslant C f(u),\left|\alpha x_{1}+\cdots+\alpha_{n} x_{n}-x_{n+1}\right| \leqslant \theta\right\}
$$

не содержит ненулевых целочисленных точек. Используя теорему Минковского о выпуклом теле, получаем

$$
2^{s} \geqslant \operatorname{mes} U(\gamma) \underset{n}{\gg}(C f(u))^{n} \theta \geqslant(C f(u))^{n} R^{-1} .
$$

ЗАмЕчАниЕ 3.1. По-видимому, справедлива следующая асимптотическая формула:

$$
\frac{1}{N^{n}} \sum_{P_{i} \in[0, N) \cap \mathbb{Z}} \# \mathfrak{B}_{f}\left(\frac{P_{1}}{N}, \ldots, \frac{P_{n}}{N}\right) \sim \frac{\mathcal{C}_{f}}{n} \ln N, \quad N \rightarrow \infty,
$$

- аналог результата Хейльбронна о средней длине непрерывной дроби для рациональных чисел с фиксированным знаменателем. Из §2 вытекает, что

$$
\frac{1}{N^{n}} \sum_{P_{i} \in[0, N) \cap \mathbb{Z}} \# \mathfrak{B}_{f}\left(\frac{P_{1}}{N}, \ldots, \frac{P_{n}}{N}\right)=2 \cdot \frac{\# \Omega_{f}^{*}(\mathbb{Z} ; N)}{N^{n}}+O_{f}(1),
$$

где $\Omega_{f}^{*}(\mathbb{Z} ; N)$ - множество целочисленных матриц $M \in \Omega_{f}$, удовлетворяющих условию $d_{s-1}(M)=1$ и имеющих фиксированньй определитель $|\operatorname{det} M|=N$.

Перейдем теперь к рассмотрению непрерывного случая.

Лемма 3.2. Функиия $\alpha \in[0,1)^{n} \rightarrow \# \mathfrak{B}_{f}(\alpha, P)$ измерима по Лебегу.

ДокАЗАтЕльство. Применим (3.1). Если $\xi(\alpha) \neq 0$, то $\alpha_{1}, \ldots, \alpha_{s-1}, 1$ линейно зависимы над кольцом $\mathbb{Z}$. Множество таких $\alpha$ имеет меру нуль по Лебегу. Поэтому

$$
\# \mathfrak{B}_{f}(\alpha, P)=2 \varphi(\alpha) \quad \text { почти при всех } \alpha \in[0,1)^{s-1} .
$$

Осталось доказать, что функция $\varphi$ измерима по Лебегу.

Предположим, что $\Omega_{f}^{\prime}-$ множество всех возможных матриц Вороного для решеток $\Gamma_{\alpha}$, т. е.

$$
\Omega_{f}^{\prime}=\left\{X \in \Omega_{f}: \Gamma(X) \in \bigcup_{\alpha \in[0,1)^{s-1}} \Gamma_{\alpha}\right\}
$$

Определим

$$
\Omega_{f, P}^{\prime}=\left\{X \in \Omega_{f}^{\prime}: f\left(x_{11}, \ldots, x_{(s-1) 1}\right) \leqslant P\right\} .
$$


Используя (2.14), получаем

$$
\varphi(\alpha)=\sum_{S \in \mathrm{SL}_{s}(\mathbb{Z})} \chi(\alpha, S), \quad \chi(\alpha, S)= \begin{cases}1, & \text { если } T_{\alpha} \in \Omega_{f, P}^{\prime} S^{-1}, \\ 0 & \text { в противном случае }\end{cases}
$$

где матрица $T_{\alpha}$ определяется (2.20). Для любой матрицы $S \in \mathrm{SL}_{s}(\mathbb{Z})$ функция $\alpha \in[0,1)^{s-1} \rightarrow \chi(\alpha, S)$ является измеримой по Лебегу как характеристическая функция некоторого измеримого множества. Значит, $\varphi$ измерима как поточечный предел последовательности измеримых функций.

ЗАмЕчАНИЕ 3.2. Пусть $\omega-$ связное подмножество в $[0,1)^{s-1}$ с липшицевой границей $\partial \omega$. Тогда для любого $R \geqslant 1$

$$
\begin{aligned}
& \frac{\#\left(\omega \cap \Delta_{s-1}(R)\right)}{\# \Delta_{s-1}(R)}=\frac{1}{\# \Delta_{s-1}(R)} \sum_{1 \leqslant N \leqslant R} \#\left(\mathbb{Z}^{s-1} \cap(N \omega)\right) \\
& \quad=\frac{1}{\# \Delta_{s-1}(R)} \sum_{1 \leqslant N \leqslant R}\left(N^{s-1} \operatorname{mes} \omega+O_{\omega}\left(N^{s-2}\right)\right)=\operatorname{mes} \omega+O_{\omega}\left(R^{-1}\right) .
\end{aligned}
$$

Для доказательства достаточно воспользоваться, например, формулой (1.2).

Вместо $\mathfrak{B}_{f}(\alpha, P)$ будем рассматривать множество

$$
\mathfrak{B}_{f}^{\prime}(\alpha, P)=\left\{(u, v) \in \mathfrak{B}_{f}(\alpha, P):\left|L_{\alpha} u-v\right| \geqslant P^{-(n+1)}\right\} .
$$

Нам нужна оценка меры множества $\alpha$ таких, что $\mathfrak{B}_{f}^{\prime}(\alpha, P) \neq \mathfrak{B}_{f}(\alpha, P)$.

Лемма 3.3. Пусть $P, Q>1$. Определим множество $G=G(P, Q)$, состоящее из точек $\alpha \in[0,1)^{n}$, удовлетворяющих условию: для любого $\alpha \in G$ существует ненулевой челочисленный вектор $(u, v) \in \mathbb{Z}^{n} \times \mathbb{Z}$ такой, что

$$
|u|_{\infty} \leqslant P, \quad\left|L_{\alpha} u-v\right| \leqslant \frac{1}{Q} .
$$

Tогда mes $G \underset{n}{\ll} P^{n} / Q$.

ДокАЗАТЕЛЬство. Возьмем любой ненулевой набор $(u, v) \in \mathbb{Z}^{n} \times \mathbb{Z}$. Пусть $g(u, v)$ - множество таких $\alpha \in[0,1)^{n}$, которые удовлетворяют (3.5). Тогда

$$
\operatorname{mes} g(u, v) \leqslant \frac{2}{Q|u|_{\infty}} \text {. }
$$

Если набор $(u, v)$ удовлетворяет (3.5) с некоторым $\alpha \in[0,1)^{n}$, то

$$
|v| \leqslant \frac{1}{Q}+\left|L_{\alpha} u\right| \leqslant(n+1)|u|_{\infty} .
$$

Следовательно, имеем

$$
\operatorname{mes} G \leqslant \sum_{u, v} \operatorname{mes} g(u, v) \leqslant \sum_{u, v} \frac{2}{Q|u|_{\infty}} \underset{n}{\ll} \frac{P^{n}}{Q},
$$

где сумма берется по всем таким $(u, v) \in \mathbb{Z}^{n} \times \mathbb{Z}$, что $1 \leqslant|u|_{\infty} \leqslant P,|v| \leqslant$ $(n+1)|u|_{\infty}$. 
Теорема 3.2. Для любого $P>1$ справедлива асимптотическая формула $(0.4)$, в которой постоянная $\mathcal{C}_{f}$ определяется (3.2).

ДокАЗАтельство. Согласно лемме 3.3 имеем

$$
\operatorname{mes}\left\{\alpha \in[0,1)^{n}: \mathfrak{B}_{f}^{\prime}(\alpha, P) \neq \mathfrak{B}_{f}(\alpha, P)\right\}=O_{n}\left(P^{-1}\right) .
$$

Напомним, что $\mathfrak{B}^{\prime}(\alpha, P)$ определяется (3.4). Поэтому, учитывая $(0.1)$, получаем

$$
\int_{[0,1)^{n}} \# \mathfrak{B}_{f}(\alpha, P) d \alpha=\int_{[0,1)^{n}} \# \mathfrak{B}_{f}^{\prime}(\alpha, P) d \alpha+O_{f}\left(\frac{\ln P+1}{P}\right) .
$$

Рассуждая так же, как и при доказательстве (3.3), приходим к формуле

$$
\# \mathfrak{B}_{f}^{\prime}(\alpha, P)=2 \psi(\alpha) \quad \text { почти при всех } \alpha \in[0,1)^{n} \text {. }
$$

Следовательно, имеем

$$
\mathcal{E}_{f}(P)=2 \int_{[0,1)^{s-1}} \psi(\alpha) d \alpha+O_{f}\left(\frac{\ln P+1}{P}\right) .
$$

Из (2.14) вытекает соотношение

$$
\psi(\alpha)=\sum_{S \in \mathrm{SL}_{s}(\mathbb{Z})} \tilde{\chi}(\alpha, S)
$$

где

$$
\tilde{\chi}(\alpha, S)=\left\{\begin{array}{ll}
1, & \text { если } Y_{\alpha} S \in \Omega_{f, P}^{\prime \prime}, \\
0 & \text { в противном случае, }
\end{array} \quad \Omega_{f, P}^{\prime \prime}=\left\{X \in \Omega_{f, P}^{\prime}: h_{1}(X) \geqslant P^{-s}\right\},\right.
$$

множество $\Omega_{f, P}^{\prime}$ определено при доказательстве леммы 3.2 .

Согласно свойствам $\Omega_{f}$ для любой $X \in \Omega_{f, P}^{\prime \prime}$ справедливы оценки

$$
\begin{gathered}
P^{-s} \leqslant h_{1}(X)=\max _{1 \leqslant j \leqslant s}\left|h_{j}(X)\right|, \quad \rho_{1}(X) \underset{f}{\ll} \rho_{2}(X), \\
h_{1}(X) \prod_{j=2}^{s} \rho_{j}(X) \underset{f}{\ll}|\operatorname{det} X|=1 .
\end{gathered}
$$

Кроме того, $\rho_{j}(X) \neq 0$ при любом $j \geqslant 2$ (иначе $\operatorname{det} X=0$ ). Учитывая, что первые $s-1$ строк матрицы $X$ целочисленные, получаем $\rho_{j}(X) \geqslant 1$. Используя эти соотношения, нетрудно проверить, что множество $\Omega_{f, P}^{\prime \prime}$ ограниченное. Значит, множества

$$
Y_{\alpha}^{-1} \cdot \Omega_{f, P}^{\prime \prime}, \quad\left\{S \in \mathrm{SL}_{s}(\mathbb{Z}): \widetilde{\chi}(\alpha, S) \neq 0\right\}
$$

ограничены равномерно по $\alpha$. Поэтому существует такое конечное множество $\mathrm{S}_{P} \subset \mathrm{SL}_{s}(\mathbb{Z})$ (зависящее только от $P$ и $f$ ), что для любого $\alpha \in[0,1)^{s-1}$

$$
\sum_{S \in \mathrm{S}_{P}} \tilde{\chi}(\alpha, S)=\sum_{S \in \mathrm{SL}_{s}(\mathbb{Z})} \tilde{\chi}(\alpha, S)=\psi(\alpha)
$$


Пусть $\omega(S)=\left\{\alpha \in[0,1)^{s-1}: Y_{\alpha} S \in \Omega_{f, P}^{\prime \prime}\right\}$. Тогда

$$
\begin{aligned}
\int_{[0,1)^{n}} \psi(\alpha) d \alpha & =\int_{[0,1)^{s-1}}\left(\sum_{S \in \mathrm{S}_{P}} \tilde{\chi}(\alpha, S)\right) d \alpha \\
& =\sum_{S \in \mathrm{S}_{P}}\left(\int_{[0,1)^{s-1}} \tilde{\chi}(\alpha, S) d \alpha\right)=\sum_{S \in \mathrm{S}_{P}} \operatorname{mes} \omega(S) .
\end{aligned}
$$

Если множество $\omega(S)$ не пусто, то оно имеет кусочно дифференцируемую границу (так как граница $\Omega_{f}$ кусочно дифференцируемая). Возьмем любое $R>P$. Используя замечание 3.2 , получаем

$$
\operatorname{mes} \omega(S)=\frac{\#\left(\omega(S) \cap \Delta_{s-1}(R)\right)}{\# \Delta_{s-1}(R)}+O_{\omega(S)}\left(R^{-1}\right) .
$$

Согласно (3.7) и (2.24) имеем

$$
\begin{aligned}
\sum_{S \in \mathrm{S}_{P}} \frac{\#\left(\omega(S) \cap \Delta_{s-1}(R)\right)}{\# \Delta_{s-1}(R)} & =\frac{1}{\# \Delta_{s-1}(R)} \sum_{S \in \mathrm{S}_{P}} \sum_{\alpha \in \Delta_{s-1}(R)} \tilde{\chi}(\alpha, S) \\
& =\frac{1}{\# \Delta_{s-1}(R)} \sum_{\alpha \in \Delta_{s-1}(R)} \psi(\alpha)=\frac{\mathcal{C}_{f}}{2} \ln P+O_{f}(1) .
\end{aligned}
$$

Используя последнюю формулу, а также (3.8), (3.9), приходим к соотношению

$$
\int_{[0,1)^{n}} \psi(\alpha) d \alpha=\sum_{S \in \mathrm{S}_{P}} \operatorname{mes} \omega(S)=\frac{\mathcal{C}_{f}}{2} \ln P+O_{f}(1)+\sum_{S \in \mathrm{S}_{P}} O_{\omega(S)}\left(R^{-1}\right) .
$$

Константы, входящие в символы $O(\ldots)$, не зависят от $R$. Множество $\mathrm{S}_{P}$ также не зависит от $R$. Поэтому, переходя к пределу при $R \rightarrow+\infty$, получаем

$$
\int_{[0,1)^{n}} \psi(\alpha) d \alpha=\frac{\mathcal{C}_{f}}{2} \ln P+O_{f}(1) .
$$

Осталось подставить последнее соотношение в (3.6). Теорема доказана.

В заключение отметим, что аналогичным образом можно исследовать вопросы о статистических свойствах наилучших совместных приближений вещественных $\alpha=\left(\alpha_{1}, \ldots, \alpha_{n}\right)$. Например, чтобы получить асимптотическую формулу для среднего числа $f$-наилучших совместных приближений рациональных $\alpha \in \Delta_{n}(R)$, нужно подсчитать количество целочисленных матриц $M \in \Omega_{f}$, удовлетворяющих условиям

$$
|\operatorname{det} M| \in[1, R], \quad \text { НОД }\left(m_{s 1}, m_{s 2}, \ldots, m_{s s}\right)=1, \quad \prod_{i=1}^{s-1} D_{i}(M)=|\operatorname{det} M|^{s-2},
$$

где $D_{i}(M)$ - наибольший общий делитель алгебраических дополнений к элементам $i$-й строки $M$.

\section{Список литературы}

1. Y. Bugeaud, M. Laurent, "On exponents of homogeneous and inhomogeneous Diophantine approximation", Mosc. Math. J., 5:4 (2005), 747-766. 
2. А. А. Илларионов, "О статистических свойствах локальных минимумов целочисленных решеток", Далъневост. матем. журн., 12:2 (2012), 201-230.

3. G. Lochs, "Statistik der Teilnenner der zu den echten Brüchen gehörigen regelmässigen Kettenbrüche", Monatsh. Math., 65 (1961), 27-52.

4. H. Heilbronn, "On the average length of a class of finite continued fractions", Number Theory and Analysis, VEB, Berlin, 1968, 87-96.

5. J. W. Porter, "On a theorem of Heilbronn", Mathematika, 22:1 (1975), 20-28.

6. А.В. Устинов, Приложения сумм Клостермана в арифметике и геометрии, LAMBERT Academic Publishing, 2011.

7. А.А. Илларионов, "О цилиндрических минимумах целочисленных решеток", Алгебра и анализ, 24:2 (2012), 154-170; англ. пер.: А. A. Illarionov, "Cylindrical minima of integral lattices", St. Petersburg Math. J., 24:2 (2013), 301-312.

8. Дж.В. Касселс, Введение в геометрию чисел, Мир, М., 1995; пер. с англ.: J.W.S. Cassels, An introduction to the geometry of numbers, Springer-Verlag, Berlin-Heidelberg-New York, 1971.

9. A. Illarionov, "On the asymptotic distribution of integer matrices", Mosc. J. Comb. Number Theory, 1:4 (2011), 13-57.

10. Г. Ф. Вороной, Об одном обобщении алгоритма непрерывных дробей, Типография Варшавского учебного округа, Варшава, 1896.

11. Г.Ф. Вороной, Собрание сочинений в 3-x mомах, т. 1, Изд-во АН УССР, Киев, 1952.

12. Б. Н. Делоне, Д. К. Фаддеев, “Теория иррациональностей третьей степени”, Тр. Матем. ин-та им. В. А. Стеклова, 11, Изд-во АН СССР, М.-Л., 1940, 3-340.

13. Дж. В. Касселс, Рациональные квадратичные формы, Мир, М., 1982; пер. с англ.: J. W. S. Cassels, Rational quadratic forms, London Math. Soc. Monogr., 13, Academic Press, London, 1978.

14. А.В. Устинов, "K теореме Вороного о цилиндрических минимумах трехмерных решеток", Дальневост. матем. журн., 11:2 (2011), 213-221.

АНДРЕЙ АНАТОЛЬЕВИч ИЛЛАРИОНОВ

Поступило в редакцию

(Andrei A. Illarionov)

13.03.2012

Хабаровское отделение

05.04 .2013

Института прикладной математики ДВО РАН,

г. Хабаровск

E-mail: illar_a@list.ru 